\title{
BCS-to-BEC crossover from the exact BCS solution
}

\author{
G. Ortiz ${ }^{1}$ and J. Dukelsky ${ }^{2}$ \\ ${ }^{1}$ Theoretical Division, Los Alamos National Laboratory, Los Alamos, New Mexico 87545, USA \\ ${ }^{2}$ Instituto de Estructura de la Materia, CSIC, Serrano 123, 28006 Madrid, Spain
}

(Received 6 April 2005; published 21 October 2005)

\begin{abstract}
The BCS-to-BEC crossover, as well as the nature of Cooper pairs, in superconducting and Fermi superfluid media is studied from the exact ground state wave function of the reduced BCS Hamiltonian. As the strength of the interaction increases, the ground state continuously evolves from a mixed system of quasifree fermions and pair resonances (BCS), to pair resonances and quasibound molecules (pseudogap), and finally to a system of quasibound molecules (BEC). A single unified scenario arises where the Cooper-pair wave function has a unique functional form. Several exact analytic expressions such as the binding energy and condensate fraction are derived. We compare our results with recent experiments in ultracold atomic Fermi gases.
\end{abstract}

DOI: 10.1103/PhysRevA.72.043611

PACS number(s): 03.75.Ss, 02.30.Ik, 05.30.Fk, 74.20.Fg

The nature of Cooper pairs in the BCS-BEC crossover has regained attention due to the observation of a large fraction of preformed fermion pairs on the BCS side of the Feshbach resonance in ultracold atomic Fermi gases [1]. While several theoretical explanations were proposed [2], the interpretations are still controversial. The root of the controversy is understanding what represents a Cooper pair in a correlated Fermi system, a concept that has been extensively used in the literature to describe the properties and dynamics of the superconducting state but without a unique and precise definition.

This paper discusses this concept at the very foundational level and proposes a qualitatively different scenario of the BCS-BEC crossover, based on the exact solution to the Bardeen-Cooper-Schrieffer (BCS) Hamiltonian [3]. Only in the extreme Bose-Einstein condensate (BEC) limit does this new scenario and Leggett's naive ansatz [4] become identical. As we will see, the Cooper pair in a correlated Fermi system is merely a statistical concept whose manifestation and interpretation depend upon the particular measurement or physical operation performed in the system.

While the superconducting and Fermi superfluid thermodynamic states represent a mixed-system of quasifree and pair-correlated fermions, the molecular BEC which arises in the dilute and strong coupling limit has all fermions bound into pairs forming a unique macroscopic quantum state. It is by now well accepted in which sense these states represent a Bose-Einstein (BE) condensation. What defines a BE condensation in an interacting $N$-particle system is spontaneous gauge symmetry breaking, or phase coherence, of its quantum state [a concept that strictly applies in the thermodynamic limit (TL)]. Yang [5] provided a detailed mathematical characterization of this phenomenon which manifests itself as off-diagonal long-range order (ODLRO) or, equivalently, by the existence of an eigenvalue of order $N$ in a reduced density matrix $\hat{\rho}_{n}$, defined as $\hat{\rho}_{n}(k l \cdots \mid i j \cdots)$ $=\operatorname{Tr}\left[a_{k} a_{l} \cdots \hat{\rho}^{\cdots} \cdot a_{j}^{\dagger} a_{i}^{\dagger}\right](i, j, k, l, \ldots$, labeling single-particle states annihilated by bosonic and/or fermionic operators $a$ 's) where $n$ is the number of particles forming the smallest unit that condenses. ( $n=2$ electrons for BCS superconductors.)

The above definition of a BEC does not imply the naive view of a many-body state of quantum objects with identical internal wave functions. Indeed, we will argue that a currentcarrying ground state (g.s.) of a uniform superconducting or Fermi superfluid $N$-particle system is of the form

$$
\Psi\left(x_{1}, \ldots, x_{N}\right)=\mathcal{A}\left[\phi_{1}\left(x_{1}, x_{2}\right) \cdots \phi_{N / 2}\left(x_{N-1}, x_{N}\right)\right],
$$

with $x_{j}=\left(\mathbf{r}_{j}, \sigma_{j}\right)$, antisymmetrizer $\mathcal{A}$, and the pair state

$$
\phi_{\alpha}\left(x_{i}, x_{j}\right)=e^{i \mathbf{q} \cdot\left[\left(\mathbf{r}_{i}+\mathbf{r}_{j}\right) / 2\right]} \varphi_{\alpha}\left(\mathbf{r}_{i}-\mathbf{r}_{j}\right) \chi\left(\sigma_{i}, \sigma_{j}\right),
$$

where $\chi$ is a spin function of a certain symmetry, $\mathbf{q}$ is the pair center-of-mass momentum, and $\varphi_{\alpha}(\mathbf{r})$ the internal wave function which may represent either a quasimolecular resonant state or a scattering state (i.e., a mixed state), depending upon the strength of the interaction between particles.

For pedagogical reasons we will recall the one-Cooperpair problem and then address the question of what happens when we add more pairs $(\mathbf{q}=\mathbf{0}$ in the g.s. $)$. The Cooper-pair solution can be recovered from the wave function (1) by assuming that $N-2$ fermions $c_{\mathbf{k} \sigma}^{\dagger}$ are free, filling the lowest momentum states $\mathbf{k}$ (of energy $\varepsilon_{\mathbf{k}}$ ) up to the Fermi momentum $\mathbf{k}_{F}$, while only an additional fermion pair (with momenta $k>k_{F}$ ) feels an attractive $s$-wave interaction in the spin singlet channel

$$
|\Psi\rangle_{C}=\sum_{k>k_{F}} \frac{1}{2 \varepsilon_{\mathbf{k}}-E} c_{\mathbf{k} \uparrow}^{\dagger} c_{-\mathbf{k} \downarrow}^{\dagger}|F\rangle .
$$

The role of the Fermi sea $|F\rangle=\prod_{k \leqslant k_{F}} c_{\mathbf{k} \uparrow}^{\dagger} c_{-\mathbf{k} \downarrow}^{\dagger}|0\rangle$, is to Pauliblock states below the Fermi energy $\varepsilon_{F}$. Assuming that the attractive pairing interaction $G<0$ is constant around the Fermi energy, the eigenvalue $E$ is always negative implying that the Cooper pair is bound regardless of the strength of the attractive interaction. The Fermi sea is then unstable against the formation of bound pairs of electrons.

What happens when the pairing interaction also affects electrons in the Fermi sea? The answer to this question is the BCS theory whose canonical form considers a simple variational state of the form (1) with all identical internal wave functions 


$$
\hat{P}_{M}|\mathrm{BCS}\rangle=\left(\Lambda^{\dagger}\right)^{M}|0\rangle, \quad \Lambda^{\dagger}=\sum_{\mathbf{k}} \frac{v_{\mathbf{k}}}{u_{\mathbf{k}}} c_{\mathbf{k} \uparrow}^{\dagger} c_{-\mathbf{k} \downarrow}^{\dagger},
$$

where $\hat{P}_{M}$ is the projector onto the state with $M$ pairs, and $v_{\mathbf{k}}, u_{\mathbf{k}}$ are the BCS amplitudes

$$
\left(\begin{array}{l}
v_{\mathbf{k}}^{2} \\
u_{\mathbf{k}}^{2}
\end{array}\right)=\frac{1}{2}\left(1 \mp\left(\varepsilon_{\mathbf{k}}-\mu\right) / \sqrt{\left(\varepsilon_{\mathbf{k}}-\mu\right)^{2}+\Delta^{2}}\right)
$$

with $\Delta$ the gap and $\mu$ the chemical potential. Since the structure of the BCS pair is averaged over the whole system, it does not characterize a Cooper pair in the superconducting/ superfluid medium except in the extreme strong-coupling and dilute limits where all pairs are bounded and nonoverlapping. [This approximate state is not the exact ground state of the reduced BCS Hamiltonian (5).] Sometimes, the structure of the Cooper pair is associated with the pair-correlation function $\left\langle\mathrm{BCS}\left|c_{\mathbf{k} \uparrow}^{\dagger} c_{-\mathbf{k} \downarrow}^{\dagger}\right| \mathrm{BCS}\right\rangle=u_{\mathbf{k}} v_{\mathbf{k}}$ describing the pair correlation among fermions of the same pair as well as the exchange between fermions of different pairs.

What is the nature of a Cooper pair in a Fermi superfluid or superconducting medium? To address this question we will use the exact solution to the reduced BCS Hamiltonian

$$
H=\sum_{\mathbf{k}} \varepsilon_{\mathbf{k}} n_{\mathbf{k}}+\frac{G}{V} \sum_{\mathbf{k}, \mathbf{k}^{\prime}} c_{\mathbf{k} \uparrow}^{\dagger} c_{-\mathbf{k} \downarrow}^{\dagger} c_{-\mathbf{k}^{\prime} \downarrow} c_{\mathbf{k}^{\prime} \uparrow},
$$

proposed by Richardson 40 years ago $[3,6]$. $H$ involves all terms with time-reversed pairs $(\mathbf{k} \uparrow,-\mathbf{k} \downarrow)$ from a contact interaction. It is consistent with an effective single-channel description of the BCS-BEC crossover theory [4] in terms of a zero-range potential. Realistic finite-range interactions produce qualitatively similar results along the crossover [7]. The main differences are expected in the BEC region where the reduced BCS Hamiltonian (5), cannot account for the reduction in the scattering length of the composite pairs [8] and the corresponding depletion of the molecular condensate [9].

For simplicity we will consider $N=N_{\uparrow}+N_{\downarrow}$ spin-1/2 (i.e., two flavor) fermions in a three-dimensional box of volume $V$ with periodic boundary conditions, interacting through an attractive constant ( $s$-wave-singlet-pairing) potential. (Other pairing symmetries can also be accommodated.) Exact $N$ $=2 M+\nu$ particle eigenstates of $H$ can be written as

$$
|\Psi\rangle=\prod_{l=1}^{M} S_{l}^{+}|\nu\rangle, \text { with } S_{l}^{+}=\sum_{\mathbf{k}} \frac{1}{2 \varepsilon_{\mathbf{k}}-E_{l}} c_{\mathbf{k} \uparrow}^{\dagger} c_{-\mathbf{k} \downarrow}^{\dagger},
$$

where $|\nu\rangle \equiv\left|\nu_{1}, \nu_{2}, \ldots, \nu_{L}\right\rangle$ is a state of $\nu$ unpaired fermions $\left(\nu=\Sigma_{\mathbf{k}} \nu_{\mathbf{k}}\right.$, with $\left.\nu_{\mathbf{k}}=1,0\right)$ defined by $c_{-\mathbf{k}} c_{\mathbf{k} \uparrow}|\nu\rangle=0$, and $n_{\mathbf{k}}|\nu\rangle=\nu_{\mathbf{k}}|\nu\rangle . L$ is the total number of single particle states. The g.s. $\left|\Psi_{0}\right\rangle$ is in the $\nu=0\left(N_{\uparrow}=N_{\downarrow}\right)$ sector.

Each eigenstate $|\Psi\rangle$ is completely defined by a set of $M$ (in general, complex) spectral parameters (pair energies) $E_{l}$ which are a solution of the Richardson's equations

$$
1+\frac{G}{V} \sum_{\mathbf{k}} \frac{1-\nu_{\mathbf{k}}}{2 \varepsilon_{\mathbf{k}}-E_{l}}+\frac{2 G}{V} \sum_{m(\neq l)=1}^{M} \frac{1}{E_{l}-E_{m}}=0,
$$

and the eigenvalues of $H$ are $\mathcal{E}=\sum_{\mathbf{k}} \varepsilon_{\mathbf{k}} \nu_{\mathbf{k}}+\sum_{l=1}^{M} E_{l}[6,10]$. A crucial observation is that if a complex $E_{l}$ satisfies Eq. (7), its complex-conjugate $E_{l}^{*}$ is also a solution. Thus, $|\Psi\rangle$ restores time-reversal invariance. The ansatz (6) is a natural generalization of the Cooper-pair problem without an inert Fermi sea, and with all pairs subjected to the pairing interaction. The pair structure in Eq. (6) is similar to the Cooper pair in Eq. (3), and the many-body state has the form (1) with all pairs different.

Since we are concerned with uniform bulk Fermi systems, we are interested in the TL (i.e., $N, V \rightarrow \infty$ with $\rho=N / V$ $=k_{F}^{3} / 3 \pi^{2}=$ const) of the Richardson's exact solution (7). This limit was studied by Gaudin [11] when the energy spectrum $\Omega \in[-\omega, \omega]$ is bounded, and $\nu=0$. Eqs. (7) reduce to the well-known gap equation

$$
\frac{1}{2} \int_{\Omega} d \varepsilon \frac{g(\varepsilon)}{\sqrt{(\varepsilon-\mu)^{2}+\Delta^{2}}}+\frac{1}{G}=0,
$$

where $g(\varepsilon)$ represents the density of states. In our case $\Omega$ $\in[0, \infty]$ is unbounded with $g(\varepsilon)=m^{3 / 2} \varepsilon^{1 / 2} /\left(\sqrt{2} \pi^{2} \hbar^{3}\right)$ for $\varepsilon_{\mathbf{k}}$ $=\hbar^{2} k^{2} / 2 m$. Due to the absence of an upper cutoff, Eq. (8) is singular and the TL becomes a subtle mathematical procedure whose solution will be presented here [12].

There are two ways to regularize the problem: One can either introduce an energy cutoff in the integrals or one can cancel the singularities with physical quantities whose bare counterpart diverges in the same way [4]. For this problem, Eq. (5), the physical quantity is the scattering length $a_{s}$ given by

$$
\frac{m}{4 \pi \hbar^{2} a_{s}}=\frac{1}{G}+\frac{1}{2} \int_{0}^{\infty} d \varepsilon \frac{g(\varepsilon)}{\varepsilon} .
$$

The nonsingular gap equation (after integration [13]) is

$$
\frac{1}{k_{F} a_{s}}=\eta=\sqrt[4]{\mu^{2}+\Delta^{2}} P_{1 / 2}\left(-\frac{\mu}{\sqrt{\mu^{2}+\Delta^{2}}}\right)
$$

where energies are now in units of $\varepsilon_{F}=\hbar^{2} k_{F}^{2} / 2 m$ and lengths in units of $\xi_{F}=1 / k_{F} . P_{\beta}(x)$ is the Legendre function of the first kind of degree $\beta$. The equations for the conservation of the number of pairs $M$

$$
-\frac{4}{3 \pi}=\eta \mu+\left(\mu^{2}+\Delta^{2}\right)^{3 / 4} P_{3 / 2}\left(-\frac{\mu}{\sqrt{\mu^{2}+\Delta^{2}}}\right),
$$

and g.s. energy density, for arbitrary strength $\eta$,

$$
\mathcal{E}_{0}=\frac{1}{V} \frac{\left\langle\Psi_{0}|H| \Psi_{0}\right\rangle}{\left\langle\Psi_{0} \mid \Psi_{0}\right\rangle}=-\frac{k_{F}^{3}}{20 \pi}\left[\frac{\eta \Delta^{2}}{2}-\frac{4}{\pi} \mu\right],
$$

do not need regularization, these are exact results. Indeed, for a given $\eta$, the chemical potential $\mu$ and gap $\Delta$ need to be determined self-consistently from Eqs. (10) and (11) (Fig. 1). Then, the g.s. energy can be computed as a function of density using Eq. (12). It shows no phase segregation. The exact binding (or condensation) energy per electron pair $\mathcal{E}_{B}$ is (see Table I) 


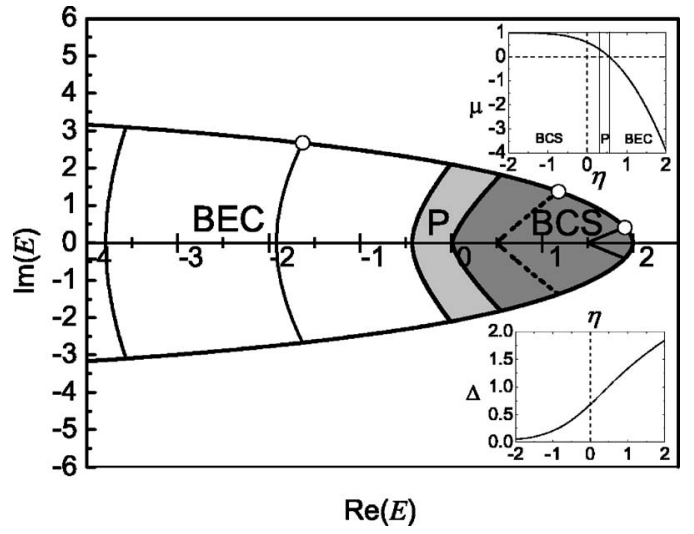

FIG. 1. Ground-state crossover diagram displaying the different regimes as a function of $\eta$. A few arcs $\Gamma$, from Eq. (14), corresponding to the values of $\eta=-1,0,0.37,0.55,1$, and 2 , whose extremes are $E_{F}=2(\mu \pm i \Delta)$, are displayed. The dashed line corresponds to the Feshbach resonance $\eta=0$. Open circles represent the values of $E$ for which Cooper-pair wave functions are plotted in Fig. 3. Insets show the behavior of the gap $\Delta$ and chemical potential $\mu$.

$$
\begin{aligned}
\mathcal{E}_{B} & =-\frac{2}{\rho}\left[\mathcal{E}_{0}-\frac{1}{V} \frac{\langle F|H| F\rangle}{\langle F \mid F\rangle}\right]=-\frac{2}{\rho}\left[\mathcal{E}_{0}-\frac{k_{F}^{3}}{5 \pi^{2}}\right] \\
& =\frac{3 \pi}{10}\left[\frac{\eta \Delta^{2}}{2}-\frac{4}{\pi}(\mu-1)\right] .
\end{aligned}
$$

The complete solution of Eqs. (7) in the TL [11] amounts to determining (for a given $\eta$ ) the set of pair energies $E_{\ell}$ which, for the g.s., form a single open, continuous, and reflection-symmetric arc $\Gamma$ with extreme points $E_{F}$ $=2(\mu \pm i \Delta)$. Here, we simply present the results without derivation. The equation for $\Gamma$ is

$$
\begin{aligned}
0 & =\operatorname{Re}\left[\int _ { 0 } ^ { \infty } d \varepsilon \sqrt { \varepsilon } \left(\frac{z+(\varepsilon-\mu) \ln \left[\frac{(E-\mu)+z}{i \Delta}\right]}{\sqrt{(\varepsilon-\mu)^{2}+\Delta^{2}}}\right.\right. \\
& \left.-\ln \left[\frac{\Delta^{2}+(E-\mu)(\varepsilon-\mu)+z \sqrt{(\varepsilon-\mu)^{2}+\Delta^{2}}}{i \Delta(\varepsilon-E)}\right]\right),
\end{aligned}
$$

where $z=\sqrt{(E-\mu)^{2}+\Delta^{2}}$.

Figure 1 shows the BCS-BEC crossover diagram and several arcs corresponding to particular values of $\eta$. Since a crossover diagram is not a phase diagram, i.e., there is no symmetry order parameter or nonanalyticities sharply differentiating the regions, boundaries are in principle arbitrary.

Here we have adopted the following criteria: The geometry of the $\operatorname{arcs} \Gamma$ serves us to establish a criterium for defining boundary regions in the crossover diagram. In the extreme weak-coupling limit, $\eta \rightarrow-\infty$, the pair energies are twice the single particle energies $\left(E_{l} \rightarrow 2 \varepsilon_{\mathbf{k}}\right)$. As soon as the interaction is switched on, i.e., $k_{F} a_{s}$ is an infinitesimal negative number, a fraction $f$ of the pair energies close to $2 \mu$ become complex, forming an arc $\Gamma$ in the complex plane. The fraction $1-f$ of fermion pairs below the crossing energy
TABLE I. Analytic expressions for selected values of $\eta$ $=1 / k_{F} a_{s} ; x \approx-0.65222953$ is the root of $P_{1 / 2}$, i.e. $P_{1 / 2}(x)=0$, and $E(y)$ is the complete elliptic integral of the second kind. Note that $5 \pi^{2} \mathcal{E}_{0}(\eta=0) / k_{F}^{3}=\mu(\eta=0) \approx 0.590606$.

\begin{tabular}{ccccc}
\hline \hline$\eta$ & $\mu$ & $\Delta$ & $\mathcal{E}_{B}$ & $\lambda$ \\
\hline$\rightarrow-\infty$ & $1+\left(\frac{\pi}{8} \eta-\frac{5}{8}\right) \Delta^{2}$ & $\frac{8}{e^{2}} \exp \frac{\pi \eta}{2}$ & $\frac{3}{4} \Delta^{2}$ & $\frac{3 \pi}{e^{2}} \exp \frac{\pi \eta}{2}$ \\
0 & $-\frac{x}{E\left(\frac{1-x}{2}\right)^{2 / 3}}$ & $\frac{\sqrt{1-x^{2}}}{E\left(\frac{1-x}{2}\right)^{2 / 3}}$ & $\frac{6}{5}\left(1+\frac{x}{E\left(\frac{1-x}{2}\right)^{2 / 3}}\right)$ & $\frac{3 \pi}{8 \sqrt{2}} \frac{\sqrt{1+x}(1-x)}{E\left(\frac{1-x}{2}\right)}$ \\
$\frac{8 \pi^{2 / 3}}{\Gamma\left[\frac{1}{4}\right]^{8 / 3}}$ & 0 & $\sqrt{2 \eta}$ & $\frac{6}{5}\left(1+\frac{\pi \eta^{2}}{4}\right)$ & $\frac{3 \pi^{3 / 2}}{\sqrt{2} \Gamma\left[\frac{1}{4}\right]^{2}}$ \\
$\rightarrow+\infty$ & $-\eta^{2}$ & $\sqrt{\frac{16 \eta}{3 \pi}}$ & $2 \eta^{2}$ & 1 \\
\hline \hline
\end{tabular}

$2 \varepsilon_{c}$ of $\Gamma$ with the real axis have real pair energies. They are still in a sea of uncorrelated pairs with an effective Fermi energy $\varepsilon_{c}$. The dark grey region labeled BCS, which extends from $\eta=-\infty$ to $\eta=0.37$, is characterized by a mixture of complex pair energies with a positive real part and real pair energies. $\eta=0.37$ is the value at which all pair energies are complex, i.e., $f=1$, and the effective Fermi sea has disappeared $\left(\varepsilon_{c}=0\right)$. Within the BCS region we plotted the arcs for $\eta=-1$ (solid line), with a fraction $f=0.35$ and an effective Fermi energy $\varepsilon_{c}=0.747$, and $\eta=0$ (dashed line) with $f$ $=0.87$ and $\varepsilon_{c}=0.25$. The pseudogap region $P$, indicated in light grey, extends from $\eta=0.37$ to $\eta=0.55$, where $\mu=0$. Within this region the real part of the pair energies changes from positive to negative, and $P$ describes a mixture of Cooper resonances and quasibound molecules. The BEC (white) region, $\eta>0.55$, is characterized by all pair energies having negative real parts, i.e., all pairs are quasibound molecules. Within the BEC region we plotted two arcs with $\eta=1$ and $\eta=2$. As $\eta$ increases further, $\Gamma$ tends to an almost vertical line with $\operatorname{Re}(E) \sim 2 \mu$, and $-2 \Delta \leqslant \operatorname{Im}(E) \leqslant 2 \Delta$.

If the literature is not clear about the size $\xi$ of a Cooper pair, it is partly because it is not clear what a Cooper pair is. (All lengths are written in units of $\xi_{F}=1 / k_{F}$.) Pippard, in his nonlocal electrodynamics of the superconducting state, introduced the characteristic length $\xi_{0}$ by using an uncertaintyprinciple argument involving only electrons within a shell of width $2 \Delta$ around the Fermi energy. The resulting quantity, named Pippard's coherence length $\xi_{0}=2 /(\pi \Delta)$ is usually associated to $\xi$. On the other hand, an acceptable definition could be $\xi=\sqrt{\left\langle\varphi\left|r^{2}\right| \varphi\right\rangle},\langle\varphi \mid \varphi\rangle=1$. From Eq. (6), the Cooperpair wave function is

$$
\varphi_{E}(\mathbf{r})=\frac{1}{V} \sum_{\mathbf{k}} \varphi_{\mathbf{k}}^{E} e^{i \mathbf{k} \cdot \mathbf{r}}=A \frac{e^{-r \sqrt{-E / 2}}}{r}
$$

with $A^{2}=\operatorname{Im}(\sqrt{E / 2}) / 2 \pi \xi_{F}^{3}, \varphi_{\mathbf{k}}^{E}=C /\left(2 \varepsilon_{\mathbf{k}}-E\right)$, and $C$ being a normalization constant. Thus, $\xi_{E}=1 / \operatorname{Im}(\sqrt{E})$. In the weakcoupling BCS limit $(\Delta \ll \mu \approx 1)$, when $E=E_{F}$, we get $\xi_{E}$ $=\pi \xi_{0} / \sqrt{2}$. On the other hand, in the same limit, if one uses $\varphi_{\mathbf{k}}^{P}=C_{P} u_{\mathbf{k}} v_{\mathbf{k}}$, one gets $\xi_{P}=\xi_{E} / 2$, and if one uses $\varphi_{\mathbf{k}}^{\mathrm{BCS}}$ $=C_{\mathrm{BCS}} v_{\mathbf{k}} / u_{\mathbf{k}}$, one gets $\xi_{\mathrm{BCS}}=\sqrt{21 / 2}$. 


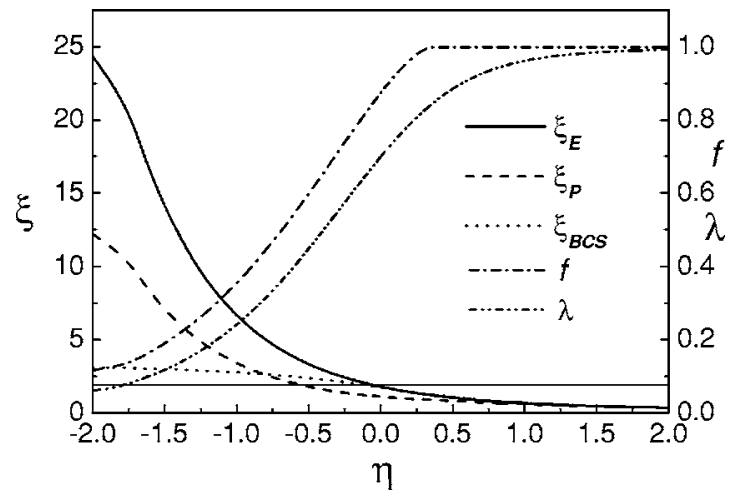

FIG. 2. Different definitions of Cooper-pair sizes $\xi$, and condensate fraction $\lambda$ and $f$ as functions of the interaction strength $\eta$. The horizontal solid line represents the interparticle distance $r_{s}$ $=\sqrt[3]{9 \pi / 4}$ (lengths are written in units of $\left.\xi_{F}=1 / k_{F}\right) . \xi_{E}$ and $\xi_{P}$ increase for negative $\eta$ values, and eventually diverge when $\eta \rightarrow-\infty$ as the inverse of the gap, similar to Pippard's coherence length $\xi_{0}$ $=2 /(\pi \Delta)$.

Analytic expressions (using dimensional regularization) for $\xi_{P}$ and $\xi_{\mathrm{BCS}}$ can also be obtained for arbitrary coupling strength. Figure 2 displays the different sizes, all labeled by $\xi$, as a function of $\eta$. Clearly, in Eq. (6) there is no unique pair size $\xi_{E}$ but a distribution of sizes; Fig. 2 shows in solid line the smallest size in $\Gamma$ corresponding to $E_{F}$. On the other hand, there is a unique pair size for $\varphi_{P}$ and $\varphi_{\mathrm{BCS}}$; the dashed and dotted lines correspond to the $\xi_{P}$ and $\xi_{\mathrm{BCS}}$ sizes, respectively. Notice the relation between sizes and the interparticle distance $r_{s}=\sqrt[3]{9 \pi / 4}$ which is indicated as a full horizontal line. While $\xi_{E}$ and $\xi_{P}$ increase for negative $\eta$ values, and eventually diverge when $\eta \rightarrow-\infty, \xi_{\mathrm{BCS}}$ tends to a constant value, related to the interparticle distance, showing its essentially uncorrelated nature. The fact that $\xi_{P}<\xi_{E} / 2$ in the overlapping BCS region is a clear demonstration that $\xi_{P}$ measures the mean distance between time-reversed pairs irrespective of the Cooper pair they belong to. For nonoverlapping pairs (BEC region) both sizes converge to the same values.

Following Yang [5], ODLRO in $\hat{\rho}_{2}\left(\mathbf{r}_{1} \uparrow \mathbf{r}_{2} \downarrow \mid \mathbf{r}_{3} \uparrow \mathbf{r}_{4} \downarrow\right)$ $\rightarrow \phi^{*}\left(\mathbf{r}_{1} \uparrow, \mathbf{r}_{2} \downarrow\right) \phi\left(\mathbf{r}_{3} \uparrow, \mathbf{r}_{4} \downarrow\right)$ may be used to define

$$
\lambda=\frac{2}{N} \int d \mathbf{r}_{1} d \mathbf{r}_{2}\left|\phi\left(\mathbf{r}_{1} \uparrow, \mathbf{r}_{2} \downarrow\right)\right|^{2}=\frac{3 \pi}{16} \frac{\Delta^{2}}{\operatorname{Im}(\sqrt{\mu+i \Delta})}
$$

as a measurement of the condensate fraction. Figure 2 shows $\lambda$ and the fraction $f$ of (Cooper) pairs in the arc, that is, the fraction of complex spectral parameters. Although $\lambda$ can qualitatively describe the fraction of correlated pairs, the fraction $f$ gives the value 1 at the BCS-pseudogap boundary $(\eta=0.37)$ while $\lambda=1$ for $\eta \rightarrow \infty$. We note that at resonance $(\eta=0), f=87 \%$ of the fermions form Cooper pairs $(\lambda \approx 0.7)$. These results provide a qualitative explanation of the experiments in Ref. [1] without resorting to a projection method [2]. Close to resonance on the BCS side, a fraction $f$ $\sim 80 \%$ of the atoms form Cooper pairs with sizes comparable to $r_{s}$. Those atom pairs are efficiently transformed into quasimolecules during a rapid magnetic field ramping across

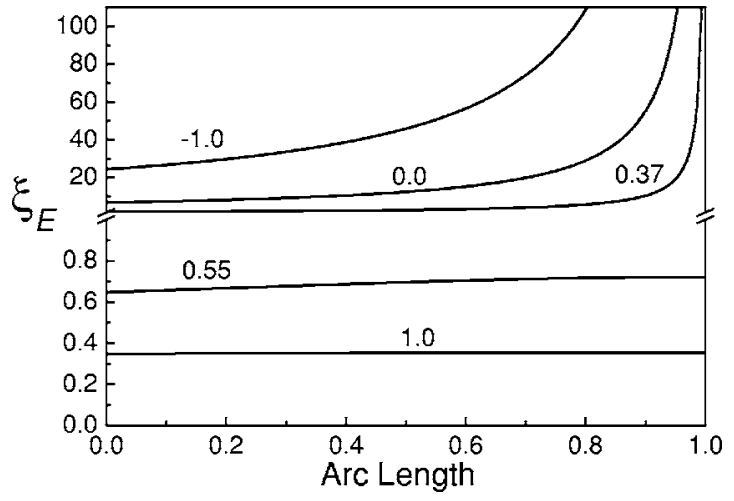

FIG. 3. Cooper-pair sizes $\xi_{E}$ along the arcs $\Gamma$ for the values of $\eta$ depicted in Fig. 1.

the resonance giving rise to the molecular condensate fractions observed experimentally.

One may argue that selecting the smallest pair from each arc to compare (in Fig. 2) Cooper-pair sizes along the crossover might not be representative of the Cooper-pair distribution within each arc. In Fig. 3 we show the internal variations of the pair sizes within the arcs. Although in the BCS region there is a distribution of sizes from the smallest pair with $E=E_{F}$ in the extremes of the arc to an almost infinite size pairs when they are close to the real axis, already at resonance $(\eta=0)$ most of the pairs have the same structure. This

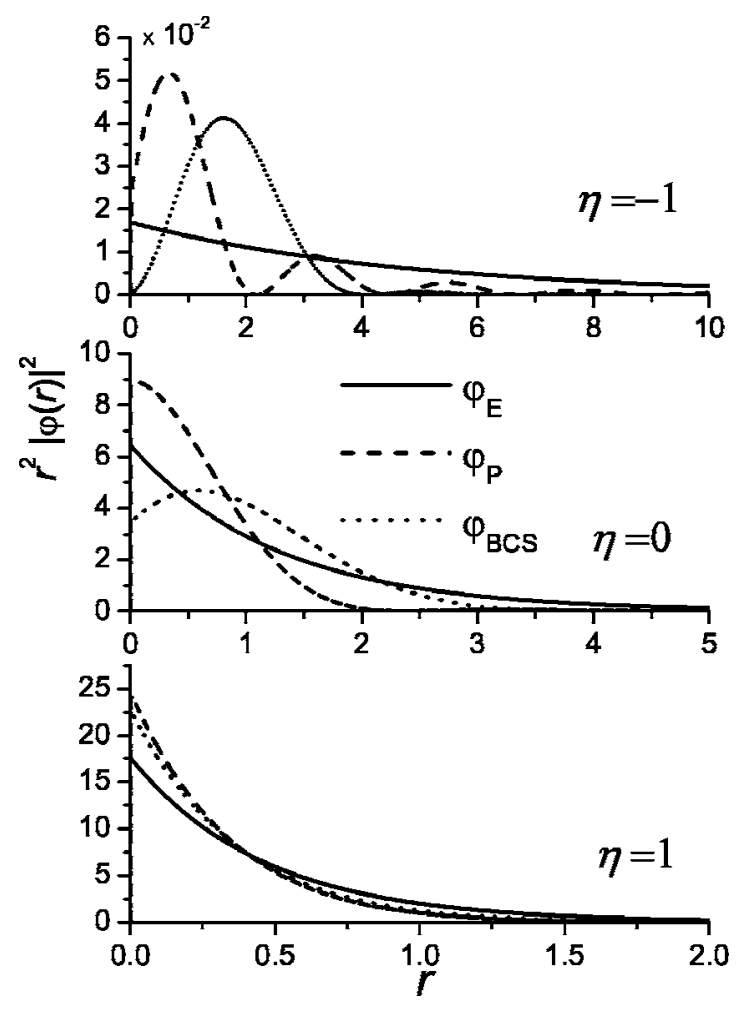

FIG. 4. Cooper-pair wave functions for different $\eta$. The upper and middle (Feshbach resonance) panels correspond to the BCS region, while the bottom one is in the BEC region. Except for the Cooper $\varphi_{E}$ case, the other two wave functions always vanish at $r$ $=0$. It is only in the limit $\eta \rightarrow+\infty$ that the three states exactly coincide. 
latter feature becomes more pronounced in the BEC region.

The various definitions of Cooper-pair wave functions are depicted in Fig. 4, which compares $\varphi_{\mathrm{BCS}}(r), \varphi_{P}(r)$, and $\varphi_{E}(r)$ for interaction strengths which correspond to the BCS, Feshbach resonance, and BEC regions of Fig. 1. Notice that while $\varphi_{\mathrm{BCS}}$, and $\varphi_{P}$ are highly oscillatory in the weakcoupling region, this is not the case with $\varphi_{E}$ which always has an exponential form. Clearly, a single and unified picture emerges for the crossover when using a many-body state such as Eq. (1): $\varphi_{E}$ evolves smoothly through the crossover as it should. It is important to mention that the three wave functions are exactly the same in the strong coupling limit $\eta \rightarrow+\infty$. In this limit the naive ansatz of Leggett [4] and the g.s. (1) coincide, becoming a simple Pfaffian state. Finally, Table I summarizes various analytical results for the chemical potential, gap, binding (or condensation) energy, and condensate fraction, in four important limits of the singlechannel model: $\eta \rightarrow-\infty$ (BCS), $\eta=0$ (resonance), $\mu=0$ (BCS-to-BEC crossover), and $\eta \rightarrow \infty$ (extreme BEC limit).

In summary, we studied the BCS-BEC crossover problem, as well as the nature of Cooper pairs in a correlated Fermi system, from the exact g.s. $\left|\Psi_{0}\right\rangle$ of the reduced BCS Hamiltonian. We have analytically determined its exact TL for the quadratic single-particle dispersion, and calculated several quantities of physical interest, such as the binding (or condensation) energy and the condensate fraction. The fundamental difference between the BCS state and $\left|\Psi_{0}\right\rangle$ is the fact that the former, being a mean field, pairs off all electrons in a unique paired wave function while the exact state contains a statistical distribution of quasimolecular resonant and scat- tering states (i.e., a mixed state) that depends upon the strength of the interaction between particles. It is only in the extreme BEC regime that the two pictures coincide asymptotically. Then, what a Cooper pair is depends upon the particular measurement or operation performed in the physical system. The validity of the present description is not restricted to integrable pairing Hamiltonians, but rather the ansatz $\left|\Psi_{0}\right\rangle$, which is a natural extension of the Cooper problem, could be used as a variational state for more realistic interactions. The Cooper-pair wave function $\varphi_{E}$ has a fixed functional $s$-wave form that accommodates pair-correlated fermions close to the Fermi energy in the weak coupling limit as well as quasibound molecules in the BEC limit. It is free from the characteristic oscillations displayed by $\varphi_{\mathrm{BCS}}$ and $\varphi_{P}$ related to exchange among pairs. The BCS region in the crossover diagram represents a mixture of Cooper pairs and quasifree fermions. It may be argued that the singlechannel model is insufficient to describe the system close to resonance where the degrees of freedom associated to the molecular closed channel should be explicitly incorporated. A derivation analogous to the one presented here can be pursued by using a recently proposed atom-molecule integrable model [14] which captures the essential features of the twochannel model [15]. The structure of the Cooper pair in this new model is the same as in Eq. (6).

We acknowledge discussions with G. G. Dussel, J. Gubernatis, A. J. Leggett, D. Loss, G. Sierra, E. M. Timmermans, and S. A. Trugman. J.D. acknowledges financial support from the Spanish DGI under Grant No. BFM2003-05316C02-02.
[1] C. A. Regal, M. Greiner, and D. S. Jin, Phys. Rev. Lett. 92, 040403 (2004); M. W. Zwierlein et al., ibid. 92, 120403 (2004); 94, 180401 (2005).

[2] R. B. Diener and T-L. Ho, cond-mat/0404517 (unpublished); A. Perali, P. Pieri, and G. C. Strinati, Phys. Rev. Lett. 95, 010407 (2005); L. Salasnich et al., cond-mat/0506074.

[3] R. W. Richardson, Phys. Lett. 3, 277 (1963); Nucl. Phys. 52, 221 (1964).

[4] D. M. Eagles, Phys. Rev. 186, 456 (1969); A. J. Leggett, in Modern Trends in the Theory of Condensed Matter, edited by A. Pekalski and R. Przystawa (Springer Verlag, Berlin, 1980); P. Nozières and S. Schmitt-Rink, J. Low Temp. Phys. 59, 195 (1985); J. R. Engelbrecht, M. Randeria, and C. A. R. Sá de Melo, Phys. Rev. B 55, 15153 (1997).

[5] C. N. Yang, Rev. Mod. Phys. 34, 694 (1962).

[6] G. Ortiz et al., Nucl. Phys. B 707, 421 (2005).

[7] M. M. Parish et al., Phys. Rev. B 71, 064513 (2005).

[8] D. S. Petrov, C. Salomon, and G. V. Shlyapnikov, Phys. Rev. Lett. 93, 090404 (2004).
[9] G. E. Astrakharchik et al., cond-mat/0507483 (unpublished).

[10] J. Dukelsky et al., Rev. Mod. Phys. 76, 643 (2004).

[11] M. Gaudin, Modèles Exactement Résolus (Les Editions de Physique, Les Ulis, 1995), p. 261; J. M. Roman, G. Sierra, and J. Dukelsky, Nucl. Phys. B 634, 483 (2002).

[12] N. N. Bogoliubov, Physica (Amsterdam) 26, S1 (1960), proved that the g.s. energy per particle of Eq. (5) in the TL equals the mean-field BCS result. (We verified that the complete set of integrals of motion [6] are also equal in the TL.) There is no existing proof that g.s. (4) and (6) are the same; this is a subtle issue. As variational states they are clearly different. An open question is what observables distinguish them at the minimum of Eq. (5) in the TL.

[13] M. Marini et al., Eur. Phys. J. B 1, 151 (1998); T. Papenbrock and G. F. Bertsch, Phys. Rev. C 59, 2052 (1999).

[14] J. Dukelsky et al., Phys. Rev. Lett. 93, 050403 (2004).

[15] M. Holland et al., Phys. Rev. Lett. 87, 120406 (2001); E. Timmermans et al., Phys. Lett. A 285, 228 (2001). 